\title{
Flow cytometric significance of cellular alkaline phosphatase activity in acute myeloid leukemia
}

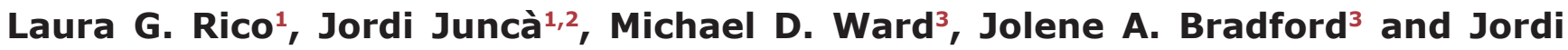 \\ Petriz $^{1}$ \\ ${ }^{1}$ Functional Cytomics Group, Institut de Recerca contra la Leucèmia Josep Carreras, IJC Campus ICO-Germans Trias i Pujol, \\ Institut Germans Trias i Pujol (IGTP), Universitat Autònoma de Barcelona, UAB, Badalona, Spain \\ ${ }^{2}$ Institut Català d'Oncologia, Hospital Germans Trias i Pujol (HGTiP), Badalona, Spain \\ ${ }^{3}$ Thermo Fisher Scientific, Eugene, Oregon, USA \\ Correspondence to: Jordi Petriz, email: jpetriz@carrerasresearch.org \\ Keywords: alkaline phosphatase; acute myeloid leukemia; stem cells; leukemic stem cells; CD34 \\ Received: August 20,2019 Accepted: November 13,2019 Published: December 10, 2019
}

Copyright: Rico et al. This is an open-access article distributed under the terms of the Creative Commons Attribution License 3.0 (CC BY 3.0), which permits unrestricted use, distribution, and reproduction in any medium, provided the original author and source are credited.

\section{ABSTRACT}

In this prospective hospital-based cohort study that included 43 newly diagnosed patients with acute myeloid leukemia, flow cytometric cellular alkaline phosphatase (ALP) activity within primitive leukemic cells allowed us to identify two groups of patients at diagnosis according to the numbers of leukemic blasts expressing $\geq 12 \%$ of ALP+ cells ( 27 patients, Group A) and less than $12 \%$ of ALP+ cells (16 patients, Group B). Differences in outcome for complete response, relapse or treatment resistance, and exitus were statistically analyzed and were significant, when comparing the two groups. The overall survival (OS) and event-free survival (EFS) differences between Group A and B were statistically significant. The survival of Group A patients was significantly shorter than those for Group B. No significant relationship was detected in outcome when comparing ELN prognostic-risk group based on cytogenetic and molecular profile (patients in the favorable, intermediate, and adverse risk groups). Flow cytometric cellular ALP activity at diagnosis may be used to estimate relapses and disease persistence more accurately. The limitations of our study include the small number of patients enrolled and a short follow-up, due to its prospective nature.

\section{INTRODUCTION}

Flow cytometry immunophenotyping [1] has become one of the mainstream applications for the diagnosis and classification of several hematologic neoplasms. This technology is indispensable for detection of leukemic blasts at a single cell level, clonal lineage assignment, identification of aberrant expression of antigens, and detection of abnormal rare populations of blasts from normal progenitors, with extreme importance in tailoring decision-making. Moreover, functional flow cytometry testing can effectively provide new insights for research and evaluation of disease, and a more complete understanding of the complexities and challenges in the analysis of leukemic stem cells.

Acute myeloid leukemia (AML) arise from stem cells, causing a rapid overproduction of abnormal myeloid blasts, usually requiring immediate treatment. AML is not a single disease and comprises a heterogeneous group of clonal hematopoietic malignancies with poor prognosis, being the most common type of acute leukemia in adults [2-4]. Patients with AML have high overall mortality rate at three years, being higher among patients 65 years or older [5]. Current strategies for the treatment of this disease, assign prognostically favorable subgroups into standard chemotherapy regimens, while unfavorable risk patients are generally considered for undergoing consolidation with allogeneic stem cell transplantation (ASCT).

Alkaline phosphatase (ALP) is expressed at very low levels in somatic cells, whereas it is highly expressed in primitive stem cells. ALP enzyme has a dimeric structure capable of binding $\mathrm{Zn}^{2+}$ and $\mathrm{Mg}^{2+}$ ions at different sites to stimulate or inhibit its catalytic 
reaction. In humans, four forms of ALP cDNA have been cloned: one of them is widely distributed (liver, bone, kidney) [6,7], one restricted to the intestine [8], one to the placenta [9], and one restricted to teratomas and germ cells [10]. ALP hydrolyzes phosphate groups and its activity is involved in a broad range of essential physiological and pathological processes. Immunohistochemical expression of ALP has been reported for embryonal carcinoma and teratoma tumors with two different alkaline phosphatases localized in stem cell populations, as well as in embryonic ectodermal cells [10]. Pluripotent stem cells, embryonic stem cells, induced pluripotent stem cells and embryonic germ cells express highly elevated ALP [11], as demonstrated by western blot, ELISA, immunohistochemistry, and highly sensitive fluorescent and chemiluminescent substrates [12].

Because stem cells lack specific cell surface markers, the identification of this compartment can be difficult, making cancer stem cells especially elusive [13]. Thus, we undertook a prospective cohort study based on our previous work [14], with the objective to investigate whether there might be differences in ALP activity within primitive leukemic cells, and its association with the potential risk of recurrence and mortality in newly diagnosed patients with AML.

\section{RESULTS}

\section{Patient characteristics and outcomes}

Between May 2015 and May 2018, a total of 106 patients were diagnosed with acute myeloid leukemia at our unit. After exclusion criteria were applied, 43 eligible patients were identified and included in the overall analysis. The characteristics of patients who were included are shown in Supplementary Table 1. The median age of patients was 67 years (range, 26-91 years). Of these, 20 (46.5\%) of patients were over 70 years of age, and 23 (53.5\%) were under 70 years of age. Overall, $30(69.8 \%)$ patients were male, and $13(30.2 \%)$ were female (Table 1). Median follow-up for OS and EFS was approximately 12 months. The 30-month OS and EFS of all AML patients was 21.3\% and $19 \%$ respectively (Figure 1A, 1B). The OS and EFS differences for adverse-, intermediate- and favorable-risk patients were also compared (Figure 1C, 1D).

\section{Alkaline phosphatase activity differences in AML: identification of two patient groups}

Differences in ALP+ blast cells at diagnosis were analyzed for the entire population. The value of ALP+ blast cells was expressed as percentages of the median and ranges around the median. Table 1 summarizes the median and range of ALP+ blast cells at diagnosis according to the age, sex, type of AML (de novo or secondary), WHO 2017 classification [15], cytogenetic and molecular alterations, European LeukemiaNet (ELN) prognostic-risk group based on cytogenetic and molecular profile [16], relevant blast immunophenotyping (CD34/ CD117/CD123 backbone), initial treatment, and postremission therapy. The diagnostic performance of the ALP test as a binary classifier system, or the accuracy of the method to discriminate two ALP+ populations, was used to determine its predictive value. As shown in Figure 2, Receiver Operating Characteristic (ROC) curve analysis (area under the curve (AUC) $=0.768,95 \%$ Confidence Interval $(\mathrm{CI})=0.596$ to $0.94, P$-value $<0.0001)$ allowed us to classify two identifiable ALP groups of patients at diagnosis according to the numbers of leukemic blasts expressing $\geq 12 \%$ of ALP + cells ( 27 patients in Group A) and less than $12 \%$ of ALP+ cells (16 patients in Group B).

\section{Differences in outcomes between groups}

Overall, 43 patients, with a median age of 63 years (range 26-91), had a median value of 18.33\% ALP+ blast cells (range 0.26-96.93). Twenty-seven patients in Group A had a median value of $23.76 \%$ of ALP+ blast cells ranging from 13.83 to 96.63 ), and 16 patients in Group B had a median value of $4.99 \%$ of ALP+ blast cells ranging from 0.26 to $11.98(P$-value $<0.0001,95 \%$ CI: $15.79-24.84)$.

Differences in outcome comparing the two groups were also analyzed. Seven patients in Group A achieved a complete response $(25.9 \%)$, in contrast with 11 patients $(68.8 \%)$ in Group B $(P$-value $=0.01,95 \% \mathrm{CI}=1.34$ to 30.99). Twenty-five patients in Group A relapsed or showed treatment resistance $(92.6 \%)$, whereas this occurred only in 5 patients $(31.3 \%)$ in Group B $(P$-value $=<0.0001,95 \%$ $\mathrm{CI}=0.00$ to 0.26$)$. Twenty-two patients in Group A died $(81.5 \%)$, whereas 5 died in Group B $(31.3 \%)(P$-value $=$ $0.002,95 \% \mathrm{CI}=0.02$ to 0.52 ). Table 2 summarizes the differences between Group A ( $\geq 12 \%$ ALP+ blasts cells) and Group B ( $<12 \%$ ALP + blasts cells) adjusted for the age, sex, type of AML (de novo or secondary), WHO Classification of AML (2017 edition), cytogenetic and molecular alterations, prognostic-risk group based on cytogenetic and molecular profile, relevant blast immunophenotyping (CD34/CD117/CD123 backbone), post-remission therapy, and outcomes (complete response achievement, relapse or treatment resistance, and exitus).

\section{Survival differences between groups}

The overall survival (OS) and event-free survival (EFS) differences between Group A and B were statistically significant. The survival for Group A patients was significantly shorter than for Group B.

The 25 -month OS in patients with $\geq 12 \%$ of ALP+ leukemic cells (Group A) was 9\%, and the 10-month EFS in the same group was $10 \%$. The 30 -month OS and EFS in patients with $<12 \%$ of ALP+ leukemic cells (Group B) was $58 \%$ and $60 \%$ respectively (Figure 3 ). 


\section{Table 1: Numbers of ALP+ blast cells at diagnosis according to patient characteristics}

\begin{tabular}{|c|c|c|}
\hline & Patients $(n=43), n(\%)$ & APL+ blast cells, median (range), $\%$ \\
\hline \multicolumn{3}{|l|}{ Age at diagnosis } \\
\hline$<70$ years & $23(53.5)$ & $13.83(1.00-96.63)$ \\
\hline$>70$ years & $20(46.5)$ & $19.86(0.26-96.63)$ \\
\hline \multicolumn{3}{|l|}{ Sex } \\
\hline - Male & $30(69.8)$ & $17.36(0.26-96.63)$ \\
\hline - Female & $13(30.2)$ & $20.75(1.62-35.91)$ \\
\hline \multicolumn{3}{|l|}{ Type of AML } \\
\hline - de novo & $30(69.8)$ & $18.11(1.20-96.63)$ \\
\hline - secondary & $13(30.2)$ & $20.75(1.00-95.92)$ \\
\hline \multicolumn{3}{|l|}{ WHO 2017 classification } \\
\hline \multicolumn{3}{|l|}{ - $A M L$ with recurrent genetic abnormalities } \\
\hline - AML with $\mathrm{t}(8 ; 21)(\mathrm{q} 22 ; \mathrm{q} 22.1) ; R U N X 1-R U N X 1 T 1$ & $2(4.7)$ & $49.17(1.71-96.63)$ \\
\hline - AML with inv(16)(p13.1q22) or $\mathrm{t}(16 ; 16)(\mathrm{p} 13.1 ; \mathrm{q} 22) ; C B F B-M Y H 11$ & $2(4.7)$ & $21.81(13.83-29.80)$ \\
\hline - AML with mutated $N P M 1$ & $8(18.6)$ & $13.85(1.00-24.71)$ \\
\hline - AML with biallelic mutations of $C E B P A$ & $3(7.0)$ & $17.09(6.53-21.75)$ \\
\hline - AML with myelodysplasia related changes & $4(9.3)$ & $19.19(9.07-95.92)$ \\
\hline - Therapy-related AML & $1(2.3)$ & $54.95(54.95-54.95)$ \\
\hline \multicolumn{3}{|l|}{ - AML not otherwise specified } \\
\hline - AML with minimal differentiation & $3(7.0)$ & $15.49(8.10-20.84)$ \\
\hline - AML without maturation & $3(7.0)$ & $22.89(2.65-23.76)$ \\
\hline - AML with maturation & $8(18.6)$ & $17.43(0.26-37.77)$ \\
\hline - Acute myelomonocytic leukemia & $4(9.3)$ & $29.10(4.96-96.63)$ \\
\hline - Acute monoblastic/monocytic leukemia & $5(11.6)$ & $18.33(1.20-26.91)$ \\
\hline \multicolumn{3}{|l|}{ Cytogenetic alterations } \\
\hline - Normal karyotype & $20(46.5)$ & $13.45(0.26-35.27)$ \\
\hline$-\operatorname{inv}(16)(p 13.1 ; q 22)$ & $2(4.7)$ & $21.81(13.83-29.80)$ \\
\hline - Monosomy 7 or del 7q & $3(7.0)$ & $23.76(15.49-54.95)$ \\
\hline - Trisomy 8 & $5(11.6)$ & $35.91(26.03-96.63)$ \\
\hline$-\mathrm{t}(8 ; 21)(\mathrm{q} 22 ; \mathrm{q} 22.1)$ & $2(4.7)$ & $49.17(1.71-96.63)$ \\
\hline$-\mathrm{t}(11 \mathrm{q} 23)$ & $1(2.3)$ & $2.65(2.65-2.65)$ \\
\hline - Complex karyotype & $3(7.0)$ & $9.53(4.96-20.84)$ \\
\hline - Others & $7(16.3)$ & $20.75(8.10-95.92)$ \\
\hline \multicolumn{3}{|l|}{ Molecular alterations } \\
\hline - NPMI mutated without FLT3-ITD & $5(11.6)$ & $5.35(1.00-17.89)$ \\
\hline - FLT3-ITD with or without $N P M 1$ mutated & $5(11.6)$ & $18.97(1.20-26.03)$ \\
\hline - FLT3-TKD & $1(2.3)$ & $29.8(29.8-29.8)$ \\
\hline - $C E B P A$ mutated & $3(7.0)$ & $17.09(6.53-21.75)$ \\
\hline - c-Kit mutated & $1(2.3)$ & $13.83(13.83-13.83)$ \\
\hline - Wild type & $28(65.1)$ & $21.56(0.26-96.63)$ \\
\hline \multicolumn{3}{|l|}{ Cytogenetic and molecular prognostic-risk group } \\
\hline - Favorable & $13(30.2)$ & $13.83(1.00-96.63)$ \\
\hline - Intermediate & $23(53.5)$ & $22.89(0.26-96.63)$ \\
\hline - Adverse & $7(16.3)$ & $9.53(1.62-26.03)$ \\
\hline \multicolumn{3}{|l|}{ Relevant LSC markers } \\
\hline - CD34+/CD123+/CD117+ & $28(65.1)$ & $20.79(0.26-96.63)$ \\
\hline - CD34+/CD123-/CD117+ & $3(7.0)$ & $95.92(2.65-96.63)$ \\
\hline - CD34-/CD123+/CD117+ & $9(20.9)$ & $9.82(1.00-30.39)$ \\
\hline - CD34-/CD123+/CD117- & $3(7.0)$ & $18.33(11.98-26.91)$ \\
\hline \multicolumn{3}{|l|}{ Initial treatment } \\
\hline - CETLAM12<70* & $20(46.5)$ & $11.82(1.00-96.63)$ \\
\hline - CETLAM12>70 & $5(11.6)$ & $15.49(0.26-35.27)$ \\
\hline - FLUGAZA clinical assay ${ }^{\ddagger}$ & $11(25.6)$ & $21.75(17.64-96.63)$ \\
\hline - Others ${ }^{\S}$ & $7(16.3)$ & $11.98(1.62-30.39)$ \\
\hline \multicolumn{3}{|l|}{ Post remission therapy } \\
\hline - Allogeneic SCT & $12(28.0)$ & $23.02(1.00-96.63)$ \\
\hline - Other & $31(72.0)$ & $17.89(0.26-96.63)$ \\
\hline
\end{tabular}

Abbreviations: APL, alkaline phosphatase; AML, acute myeloid leukemia; WHO, world health organization; NPM1, nucleophosmin 1; FLT3-ITD, fms-like tyrosine kinase 3 internal tandem duplication; FLT3-TKD, fms-like tyrosine kinase 3 tyrosine kinase domain; SCT, stem cell transplant.

"Cytarabine + idarubicin; ${ }^{\circ}$ Cytarabine + fludarabine; ${ }^{*}$ Cytarabine + fludarabine / Azacitidine; ${ }^{8}$ Azacitidine / Decitabine / Cytarabine + idarubicin + quizatinib or placebo / None. 


\section{Multivariable analysis}

Univariate and multivariate Cox proportional hazards models were used to identify factors that are associated with OS and EFS in patients with AML (Table 3). ALP expression was a significant predictor of OS on univariate (hazard ratio $[\mathrm{HR}]=0.32,95 \% \mathrm{CI}=$ 0.11 to $0.87, P$-value $<0.025)$ analysis. ALP expression was a significant predictor of EFS on univariate ([HR] $=0.19,95 \% \mathrm{CI}=0.17$ to $0.52, P$-value $=0.0012)$ and multivariate $([\mathrm{HR}]=0.25,95 \% \mathrm{CI}=0.09$ to $0.70, P$-value $<0.0079)$ analyses. In the univariate Cox model for OS, age, favorable and intermediate risk, AML type, and ALP Group were associated with a significant impact on survival. The only factor that retained their significance on multivariate analysis was age. In the univariate Cox
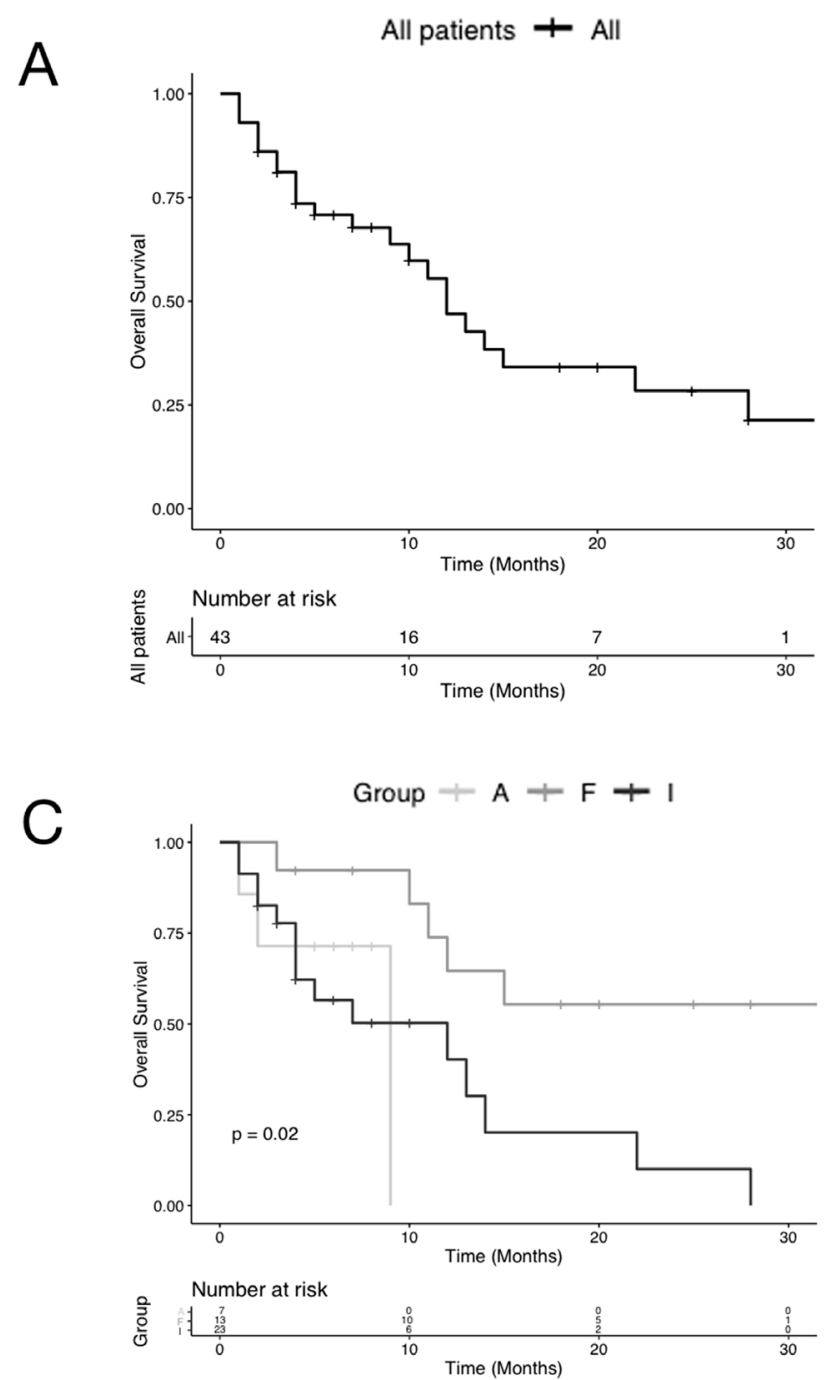

model for EFS, age, favorable and intermediate risk, and ALP Group were associated with a significant impact on progression/relapse. The only factors that retained their significance on multivariate analysis were favorable risk and ALP group.

\section{DISCUSSION}

Acute myeloid leukemia is a heterogeneous clonal disorder associated with a relatively high early mortality rate and poor overall chances for recovery. Even with current treatments, the relative 5-year survival rate for patients with AML is only 5\% after the age of 65 [17]. Current diagnosis and medical treatment of AML is expensive, not just in terms of cost, but also in terms of the impact on prolonged overall treatment duration for
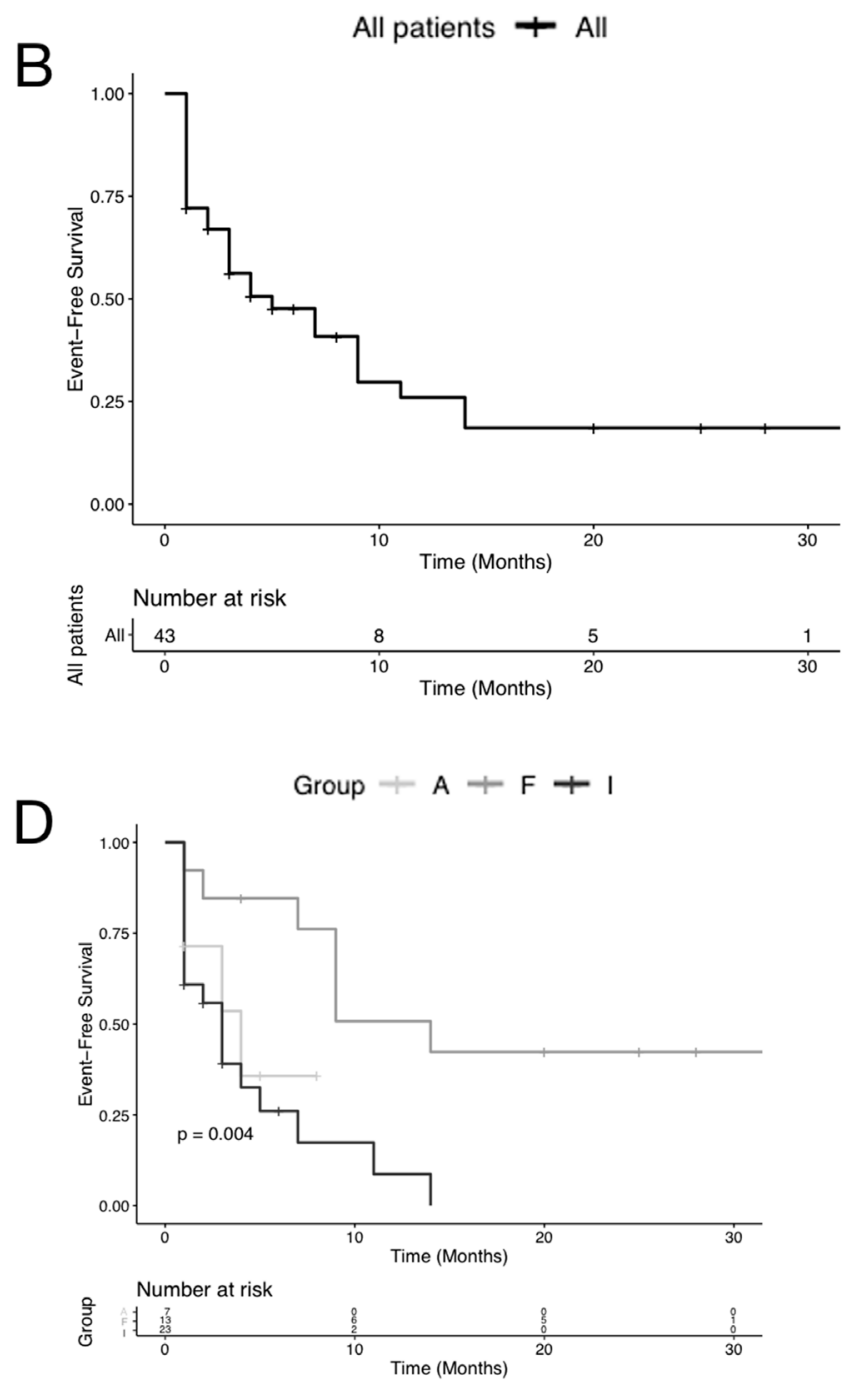

Figure 1: Plots of Kaplan-Meier limit estimates of overall survival and event-free survival curve analysis of acute myeloid leukemia patients at diagnosis. Plots of Kaplan-Meier limit estimates of overall and event-free survival of acute myeloid leukemia ungrouped and grouped patients according to the risk. Plots of Kaplan-Meier limit estimates of overall and event-free survival of acute myeloid leukemia ungrouped patients are shown in (A and $\mathbf{B})$ respectively. Overall and event-free survival differences for adverse-, intermediate- and favorable-risk patients (A, I, and F) are shown in (C and D) respectively. 
patients and their families. Despite new therapy options having been developed to treat AML, advancement in understanding the pathogenesis of AML is continuously needed. Furthermore, the capability to accurately predict survival is often difficult, raising the need for new predictive biomarkers in AML therapeutics.

The fundamental goal of personalized medicine, aimed at providing patient-specific molecular insights, combines genomic, clinical, and other types of data, to provide strategies aimed at treatment optimization based on individual patient characteristics. In recent years, advances in genotypic arrays, digital PCR, and next-generation sequencing, also known as high-throughput sequencing, have played a key role in developing new assays, such as those for predicting AML relapse after ASCT, for mutational screening, risk stratification, guided therapy, or Measurable residual disease (MRD) monitoring. Multiparameter flow cytometry in the clinical laboratory has also become an essential technology to increase the sensitivity of leukemia detection at the single cell level, especially for identifying very low levels of residual disease immediately after treatment and during follow-up.

Assessment of MRD is today considered indispensable for forecasting the significance and association with treatment outcome and survival [18].
However, the sensitivity of the assays, phenotypic shifts at relapse, low frequency of residual leukemic stem cells, quality of the marrow aspirate, or the use of peripheral blood, may lead to identifying false-positive and falsenegative patient groups by current approaches [19]. AML comprises a dynamic disease with a very complex landscape of aberrant phenotypic, cytogenetic and molecular genetic abnormalities, that importantly confer independent biological properties of the leukemic cells.

In 1970, Clarkson et al. found leukemic cells to behave as a self-maintaining population [20]. Although their data did not preclude some influx of leukemic "stem cells" from an unrecognized precursor compartment, they found no evidence to support this hypothesis. On the same subject of AML, Bonnet and Dick (1997), demonstrated that leukemic cells obtained from patients possess differentiative and proliferative capacities, as well as the self-renewal properties found in leukemic stem cells [21]. However, the heterogeneity of the stem cell compartment supports that the stem cell concept is not necessarily associated with a specific cellular entity, but rather a function that can be assumed by numerous diverse cell types. As a result, the cancer stem cell concept cannot be universally applicable, based on complex evolution, phenotypic heterogeneity and therapeutic challenges of many human cancers, making

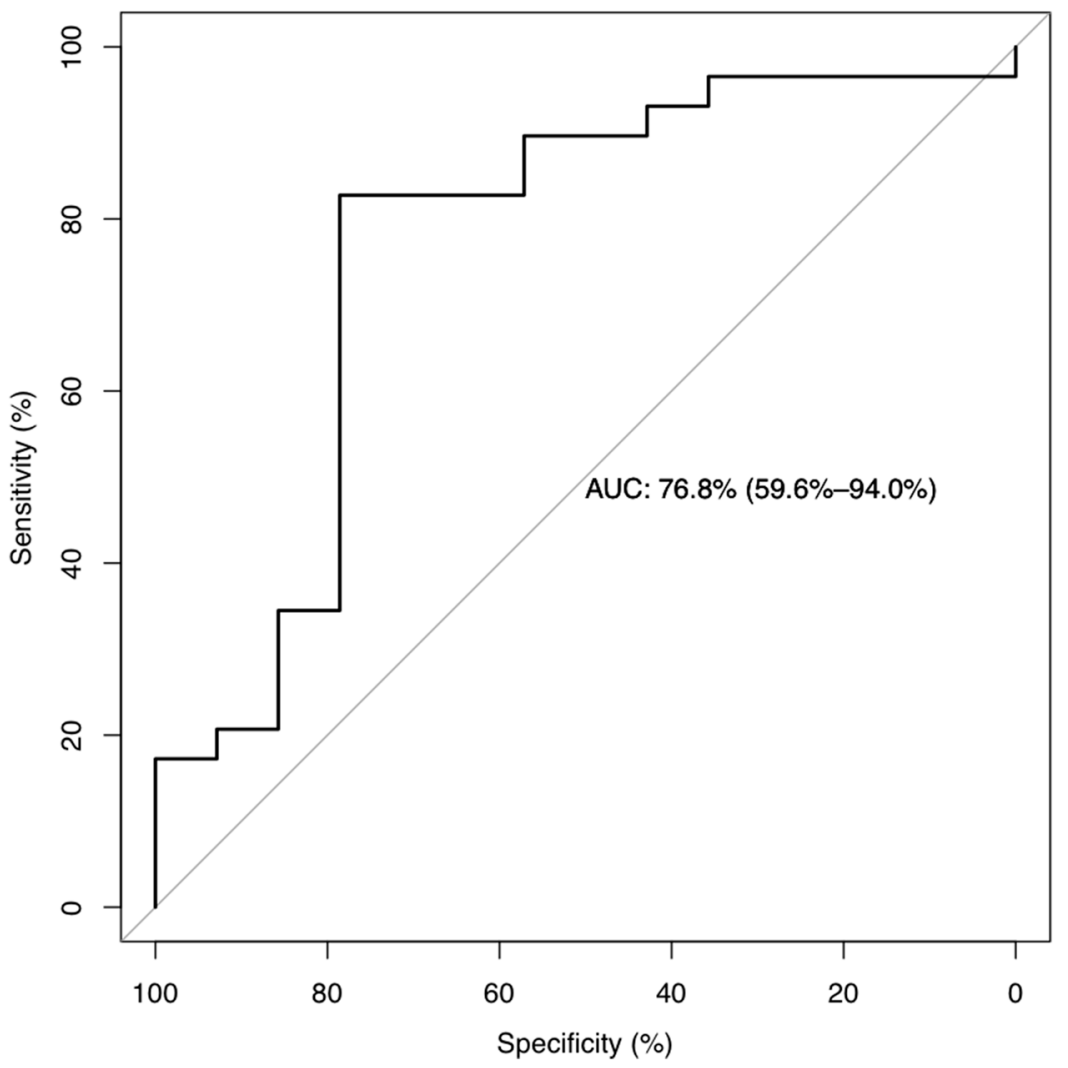

Figure 2: Receiver operating characteristic (ROC) curves. According to ROC curve analysis, $12 \%$ of ALP + was confirmed as the cut-off point of ALP+ leukemic cell counting for survival outcome of AML patients. ROC curve analysis (area under the curve $=0.768$, $95 \% \mathrm{CI}=0.596$ to $0.94, P$-value $<0.0001)$ classified two identifiable ALP groups of patients at diagnosis according to the numbers of leukemic blasts expressing $\geq 12 \%$ of ALP+ cells ( 27 patients in Group A) and less than $12 \%$ of ALP+ cells (16 patients in Group B). 
Table 2: Differences between Group A and Group B regarding total number of ALP+ blast cells at diagnosis

\begin{tabular}{|c|c|c|c|c|}
\hline & Overall $(n=43)$ & Group A $(n=27)$ & Group B $(n=16)$ & $P$-value $(95 \% \mathrm{CI})$ \\
\hline ALP+ blast cells, median (range), $\%$ & $18.33(0.26-96.63)$ & $23.76(13.83-96.63)$ & $4.99(0.26-11.98)$ & $<0.0001(15.79-24.84)^{* * * *}$ \\
\hline Age at diagnosis, median (range), years & $63.0(26-91)$ & $73.0(26-81)$ & $59.5(31-91)$ & $0.38(-5.00-17.00)$ \\
\hline \multicolumn{5}{|l|}{$\operatorname{Sex}, n(\%)$} \\
\hline - Male & $30(69.8)$ & $17(63.0)$ & $13(81.3)$ & \multirow{2}{*}{$0.31(0.50-16.99)$} \\
\hline - Female & $13(30.2)$ & $10(37.0)$ & $3(18.7)$ & \\
\hline \multicolumn{5}{|l|}{ Type of AML, $n(\%)$} \\
\hline - de novo & $30(69.8)$ & $19(70.4)$ & $11(68.8 \%)$ & \multirow{2}{*}{$1(0.22-4.93)$} \\
\hline - secondary & $13(30.2)$ & $8(29.6)$ & $5(31.2 \%)$ & \\
\hline \multicolumn{5}{|l|}{ WHO 2017 classification, $n(\%)$} \\
\hline - AML with recurrent genetic abnormalities & $15(34.9)$ & $9(33.3)$ & $6(37.5)$ & $1(0.27-5.18)$ \\
\hline - AML with myelodysplasia related changes & $4(9.3)$ & $3(11.1)$ & $1(6.3)$ & $1(0.00-7.47)$ \\
\hline - Therapy-related AML & $1(2.3)$ & $1(3.7)$ & $0(0.0)$ & $1(0.00-65.75)$ \\
\hline - AML not otherwise specified & $23(53.5)$ & $14(51.9)$ & $9(56.2)$ & $1(0.29-4.99)$ \\
\hline \multicolumn{5}{|l|}{ Molecular alterations, $n(\%)$} \\
\hline - NPM1 mutated without FLT3-ITD & $5(11.6)$ & $1(3.7)$ & $4(25.0)$ & $0.06(0.71-441.52)$ \\
\hline - FLT3-ITD with or without $N P M 1$ mutated & $5(11.6)$ & $3(11.1)$ & $2(12.5)$ & $1(0.09-11.26)$ \\
\hline - NPMI and FLT3 wild type & $33(76.8)$ & $23(85.2)$ & $10(62.5)$ & $0.14(0.05-1.58)$ \\
\hline \multicolumn{5}{|l|}{$\begin{array}{l}\text { Cytogenetic and molecular prognostic-risk } \\
\text { group, } n(\%)\end{array}$} \\
\hline - Favorable & $13(30.2)$ & $7(25.9)$ & $6(37.5)$ & $0.50(0.36-7.82)$ \\
\hline - Intermediate & $23(53.5)$ & $17(63.0)$ & $6(37.5)$ & $0.12(0.08-1.50)$ \\
\hline - Adverse & $7(16.3)$ & $3(11.1)$ & $4(25.0)$ & $0.39(0.37-20.75)$ \\
\hline Blasts at diagnosis, median (range), $\%$ & $45.0(6.0-98.0)$ & $50.0(18.0-98.0)$ & $39.0(6.0-88.8)$ & $0.35(-7.99-23.00)$ \\
\hline WBC at diagnosis, median (range), $n \times 10^{9} / \mathrm{L}$ & $8.5(0.5-249.1)$ & $5.9(0.7-249.1)$ & $13.3(0.5-65.4)$ & $0.93(-10.80-6.60)$ \\
\hline \multicolumn{5}{|l|}{ Relevant LSC markers, $n(\%)$} \\
\hline - CD34+/CD123+/CD117+ & $28(65.1)$ & $19(70.0)$ & $9(56.2)$ & $0.51(0.12-2.39)$ \\
\hline - CD34+/CD123-/CD117+ & $3(7.0)$ & $2(7.5)$ & $1(6.3)$ & $1(0.01-17.38)$ \\
\hline - CD34-/CD123+/CD117+ & $9(20.9)$ & $4(15.0)$ & $5(31.2)$ & $0.26(0.45-15.67)$ \\
\hline - CD34-/CD123+/CD117- & $3(7.0)$ & $2(7.5)$ & $1(6.3)$ & $1(0.01-17.38)$ \\
\hline \multicolumn{5}{|l|}{ Post remission therapy, $n(\%)$} \\
\hline - Allogeneic SCT & $12(28.0)$ & $8(30.0)$ & $4(25.0)$ & \multirow{2}{*}{$1.0000(0.26-7.00)$} \\
\hline - Other & $31(72.0)$ & $19(70.0)$ & $12(75.0)$ & \\
\hline \multicolumn{5}{|l|}{ Outcomes, $n(\%)$} \\
\hline - Complete response & $18(41.9)$ & $7(25.9)$ & $11(68.8)$ & $0.01(1.34-30.99)^{*}$ \\
\hline - Relapse or Treatment resistance & $30(69.8)$ & $25(92.6)$ & $5(31.3)$ & $<0.0001(0.00-0.26)^{* * * *}$ \\
\hline - Exitus & $27(62.8)$ & $22(81.5)$ & $5(31.3)$ & $0.002(0.02-0.52)^{* * *}$ \\
\hline
\end{tabular}

Abbreviations: CI, confidence interval; ALP, alkaline phosphatase; n, number; AML, acute myeloid leukemia; WHO, world health organization; NPM1, nucleophosmin 1; FLT3-ITD, fms-like tyrosine kinase 3 internal tandem duplication; WBC, white blood cells; L, liter; LSC, leukemic stem cell; SCT, stem cell transplant.

${ }^{*} p$-value $<0.05 ;{ }^{* *} p$-value $<0.01 ; * * * *$-value $<0.0001$.

difficult the identification of rare molecular and phenotypicbased putative leukemic stem cells [22, 23].

Aside from antigen expression properties on AML bulk cells, and that antigen coexpression on leukemic stem cells (LSC) makes it difficult to specifically target this compartment, LSCs have unique features based on metabolic response to drugs, cell stress control, and other key aspects of stemness and chemotherapy resistance. Based on the clonal nature of tumors, and after the demonstration that leukemic stem cells from patients 
Table 3: Multivariate analyses

\begin{tabular}{|c|c|c|c|c|c|c|c|c|}
\hline \multirow[b]{2}{*}{ Variable } & \multicolumn{4}{|c|}{ Overall Survival (OS) } & \multicolumn{4}{|c|}{ Event-Free Survival (EFS) } \\
\hline & $\begin{array}{c}\text { Univariate } \\
\text { Hazard ratio } \\
(95 \% \mathrm{CI})\end{array}$ & $P$-value & $\begin{array}{c}\text { Multivariate } \\
\text { Hazard ratio } \\
(95 \% \mathrm{CI})\end{array}$ & $P$-value & $\begin{array}{c}\text { Univariate } \\
\text { Hazard ratio } \\
(95 \% \mathrm{CI})\end{array}$ & $P$-value & $\begin{array}{c}\text { Multivariate } \\
\text { Hazard ratio } \\
(95 \% \mathrm{CI})\end{array}$ & $P$-value \\
\hline Age & $1.10(1.00-1.10)$ & $0.0014^{* *}$ & $1.04(1.00-1.08)$ & $0.0341^{*}$ & $1.00(1.00-1.10)$ & $0.039^{*}$ & $1.01(0.99-1.05)$ & 0.21 \\
\hline Sex & $1.40(0.56-3.40)$ & 0.48 & $0.58(0.24-1.92)$ & 0.47 & $1.00(0.45-2.20)$ & 1.00 & - & - \\
\hline ALP Group & $0.32(0.11-0.87)$ & $0.025^{*}$ & $0.47(0.15-1.34)$ & 0.15 & $0.19(0.07-0.52)$ & $0.0012^{* *}$ & $0.25(0.09-0.70)$ & $0.0079^{* *}$ \\
\hline AML Type & $4.20(1.80-9.80)$ & $0.0009^{* * *}$ & $1.50(0.49-5.27)$ & 0.43 & $1.60(0.71-3.50)$ & 0.27 & $0.48(0.16-1.46)$ & 0.20 \\
\hline Favorable Risk & $0.25(0.09-0.70)$ & $0.0086^{* *}$ & $0.33(0.07-1.69)$ & 0.18 & $0.24(0.09-0.61)$ & $0.0026^{\text {*** }}$ & $0.23(0.06-0.93)$ & $0.039^{*}$ \\
\hline $\begin{array}{c}\text { Intermediate } \\
\text { Risk }\end{array}$ & $2.70(1.00-5.80)$ & $0.042^{*}$ & $0.78(0.19-3.11)$ & 0.72 & $3.10(1.40-6.80)$ & $0.0053^{\text {** }}$ & $1.01(0.33-3.12)$ & 0.99 \\
\hline Adverse Risk & $1.60(0.44-5.70)$ & 0.50 & - & - & $1.20(0.41-3.60)$ & 0.72 & - & - \\
\hline$\%$ Blasts & $0.99(0.97-1.00)$ & 0.20 & $1.00(0.98-1.03)$ & 0.76 & $1.00(0.98-1.00)$ & 0.99 & - & - \\
\hline WBC & $0.99(0.98-1.00)$ & 0.46 & $1.00(0.99-1.01)$ & 0.94 & $1.00(0.99-1.00)$ & 0.64 & - & - \\
\hline
\end{tabular}

Abbreviations: CI, confidence interval; ALP, alkaline phosphatase; AML, acute myeloid leukemia; WBC, white blood cells. ${ }^{*} p$-value $<0.05 ;{ }^{* *} p$-value $<0.01 ;{ }^{* * *} p$-value $<0.001$.

with AML reconstitute the full spectrum of phenotypes in the malignant populations that they regenerate in transplanted mice, we hypothesized that ALP could help to identify primitive leukemic cells [14]. Moreover, it has been demonstrated that ALP activity may be useful for the diagnosis of acute myelomonocytic and monocytic leukemia in dogs [24]. In parallel with flow cytometric immunophenotyping, Stokol et al. used a cytochemical staining approach for ALP activity, concluding that ALP activity in canine AML can be especially helpful when flow cytometric results are inconclusive. We then decided

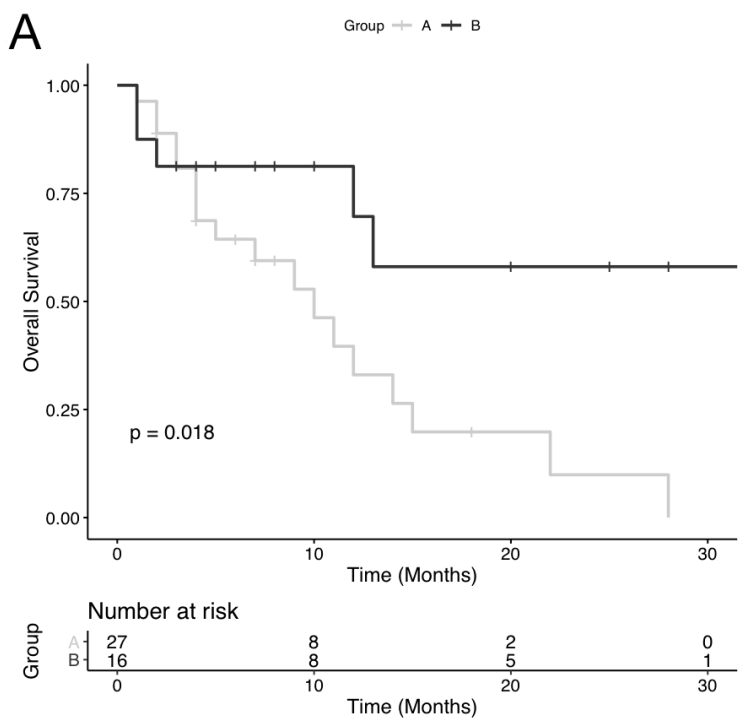

to implement a new ALP flow cytometric method test, using a novel fluorogenic live cell permeant substrate for ALP [12], since this stain efficiently visualized pluripotent stem cells such as human embryonal carcinoma, murine and human embryonic stem cells and induced pluripotent stem cells under fluorescent microscopy [25]. Our method uses a no-lyse no-wash approach, offering opportunities to combine live cell response and functional assessment in combination with cell immunophenotyping (Supplementary Figure 1), while minimizing sample preparation effects on the cell biology as the primary

Figure 3: Plots of Kaplan-Meier limit estimates of overall survival and event-free survival curve analysis of acute myeloid leukemia patients, according to the numbers of alkaline phosphatase (ALP) positive leukemic cells at diagnosis. Overall survival after diagnosis was compared between high ALP Group (A) and low ALP Group (B). Patients with ALP+ leukemic cells $\geq$ $12 \%$ achieved shorter OS than those with ALP+ leukemic cells $<12 \%$. According to ROC curve analysis, $12 \%$ of ALP+ was confirmed as the cut-off point of ALP+ leukemic cell counting for survival outcome of AML patients. Kaplan-Meier plots of overall survival for grouped acute myeloid leukemia patients are shown in (A). Plots of Kaplan-Meier product limit estimates of event-free survival of grouped patients according to the numbers of ALP positive leukemic cells at diagnosis are shown in (B). 
goal [26]. This method can also be used to identify rare cells [27]. Additionally, assays dealing with live kinetic analysis, like calcium flux and enzyme rate, or assays that depend on continued active drug transport just prior to analysis, like side population analysis must be done with live cells, preferably with as little sample manipulation as possible. Recent work by the authors [14] showed elevated ALP activity in CD34+ cells in highly refractory cancers. This study was made possible by using this new non-toxic cell-permeant fluorescent ALP for live cells without need to add multidrug resistant transporter inhibitors, taking advantage of one of the most-widely used enzyme-based standards, known as the aldehyde dehydrogenase (ALDH) assay [28]. Previously, we demonstrated measurement of ALP activity with ABCG2 efflux pump activity using this reagent in combination with side population analysis, making its use feasible for primitive stem cells expressing $\mathrm{ABC}$ transporters. This can only be done with live healthy cells at the point of analysis [14].

Measuring live cell response function and immunophenotype with the minimum possible sample manipulation, may increase the potential for discovery of clinically relevant cell subsets. This area of research is particularly interesting from a both theoretical and practical point of view. As far as we know, some phenotypic characteristics may relate to clinical outcome in AML patients, i.e, CD34 negativity related with NPM1 mutation, CD56 expression in AML with $(8 ; 21)$, or CD2, CD36, CD11b or CD56 positivity in other types of AML. However, attempts to characterize these associations have yielded inconclusive results. Given we lack specific markers to identify if a given phenotype is tightly linked or specifically caused by some mutations, maybe the only way to identify rare cell types is by combining phenotype information with and functional assays.

At the light of our results, increased cellular ALP activity in AML with no evidence of aberrant antigen expression is still unclear and should be confirmed in a large series of patients. Importantly in the low residual disease, MRD evaluation by whatever the method (leukemia-associated immunophenotypes (LAIPs) identification vs. "different from normal" approaches) has the value of indicating the clinicians "how things are going", and that it may have a definitive underlying functional importance is to achieve and maintain a low or negative value for ALP activity, because a marked increase in this biomarker could be interpreted to reflect increased pathological functional activity in a not yet detected leukemic but previously fully identified population, the emergence of a new and probably more immature and aggressive type of cells that may in fact determine the clinical course of the patient, or to provide some clues for more proliferative advantages related to a given phenotype.

In this work, we have shown and validated that ALP expression by blast cells at diagnosis may have a significant impact to identify two different groups of patients. In addition, we have demonstrated that ALP also has a significant impact to predict complete response, relapse or treatment resistance, and exitus, independently of variables such as age, sex, type of AML (de novo or secondary), WHO Classification of AML (2017 edition), cytogenetic and molecular alterations, ELN prognosticrisk group based on cytogenetic and molecular profile, relevant blast immunophenotyping (CD34/CD117/CD123 backbone), initial treatment, and post-remission therapy (Table 2).

Despite the cohort's small number, some risk factors correlated with overall survival and event free survival. Specifically, age entered all univariate and multivariate models except with the EFS in the multivariate, and favorable risk only did not correlated well with OS in the multivariate. Intermediate risk correlated well with OS and EFS in the univariate. Finally, the ALP group was significant in the univariate model for EFS and OS, as well as in the multivariate model for EFS. The ALP group only did not correlate with OS in the multivariate (Table 3).

Surprisingly, the percentage of ALP+ blast cells in the intermediate risk group (median: 22.89, range: 0.26-96.63) was higher than that of the adverse risk group (median: 9.53; range: 1.62-26.03), suggesting that the intermediate risk group may include a relative number of patients with adverse risk (Table 1). Despite that, this result can also be limited by the bias of the cohort's small number, this observation is supported by the OS and EFS Kaplan-Meier analyses according to favorable, intermediate, and adverse definitions (Figure 1C and 1D). Even with these limitations, our results have significant clinical and scientifically important implications.

The outcome of AML treatment is highly variable and still not individually predictable [29]. Currently, the most refined predictive data that relate to the prognosis of AML are those derived of cytogenetic and molecular analysis. However, other most determinant predictive factors may exist, and that they may reflect the degree of immaturity of the main burden of cells that proliferate in a given case of AML, and that this degree of immaturity (as judged by a functional analysis) is more prognostically determinant that the cytogenetic or molecular characteristics of leukemic cells. However, this is only a hypothesis to verify, and, in fact, we have no explanation for the apparently contradictory result of the lack of correlation of ALP and cytogenetics. Relative to other cancers, AML has a comparatively low level of genetic heterogeneity, suggesting that epigenetic heterogeneity is of primary importance. Thus, epigenetic factors such as DNA methylation, genomic imprinting, histone modifications, and expression control by noncoding RNA, may also play an important role. If confirmed in larger series of patients, our results open a new perspective of research in the analysis of outcome in AML. Moreover, if cellular alkaline phosphatase activity is a prognosticator 
at diagnosis, it could help to investigate how to reach the ultimate goal of individualized risk assessment as a guide for therapy decision-making, or to design new novel treatment targets in AML. The development of novel ALP inhibitors and modulators [30] for the treatment of AML are promising approaches to reduce the increased mortality associated with this heterogeneous disease characterized by a multitude of molecular abnormalities. Since several methods are only applicable for MRD detection on a clinical routine basis, cellular ALP could help to better classify or to predict the behavior of the disease, as well as to is really predicting the outcome in a given patient. Furthermore, it would be also very interesting to study the comparative value of monitoring MRD regularly with flow cytometric ALP, as truly negative non-detectable MRD status has strong clinical relevance in AML, making it an essential tool in the overall strategy adopted to treat AML.

The limitations of our study include the small number of patients enrolled and a short follow-up, due to its prospective nature. We are fully aware that standard risk assessment markers have been well validated in numerous large studies and suggest that findings in this small set of 43 patients may not be valid in a larger population. Despite this small number, this study offers evidence to the hypothesis that intrinsic cellular ALP activity at diagnosis may be used to estimate relapses and disease persistence more accurately. Larger studies will be needed to determine whether ALP activity in primitive leukemic cells is associated with the potential risk of recurrence and mortality in newly diagnosed patients with AML.

Since the discovery of the hybridoma technology, many monoclonal antibodies have been available for laboratory studies, making possible to dissect human malignancy. In the next few years, more investigations should shed light on the translational utility of new available biological indicators aimed at detecting cell function, and discovering in combination with flow cytometry immunophenotyping, the complex and heterogeneous biology of the stem cell compartment in human pathology.

\section{MATERIALS AND METHODS}

\section{Study design and patient population}

This was a prospective cohort study of patients newly diagnosed with acute myeloid leukemia and completed follow-up. From May 2015 through May 2018, 106 patients were diagnosed with AML at the hematological cytometry unit at Germans Trias i Pujol Hospital (HGTiP) in Badalona (Spain) that covers an area of approximately 700,000 inhabitants. After applying the exclusion criteria, 43 patients (male 30, female 13; median age 63 years, range 26-91) were included in our study. Most of the patients were initially treated according to the Spanish CETLAM protocols, based on the use of idarubicin or fludarabine with cytarabine. A total of 12 out of 43 patients underwent allogeneic stem cell transplant (ASCT) following chemotherapy. The patient's risk (favorable, intermediate, adverse) was evaluated according to ELN 2017 recommendations, based on cytogenetic and/or molecular abnormalities [16]. Details of patient population and statistics are provided in Table 1 and Table 2 respectively. All patients enrolled in this study provided
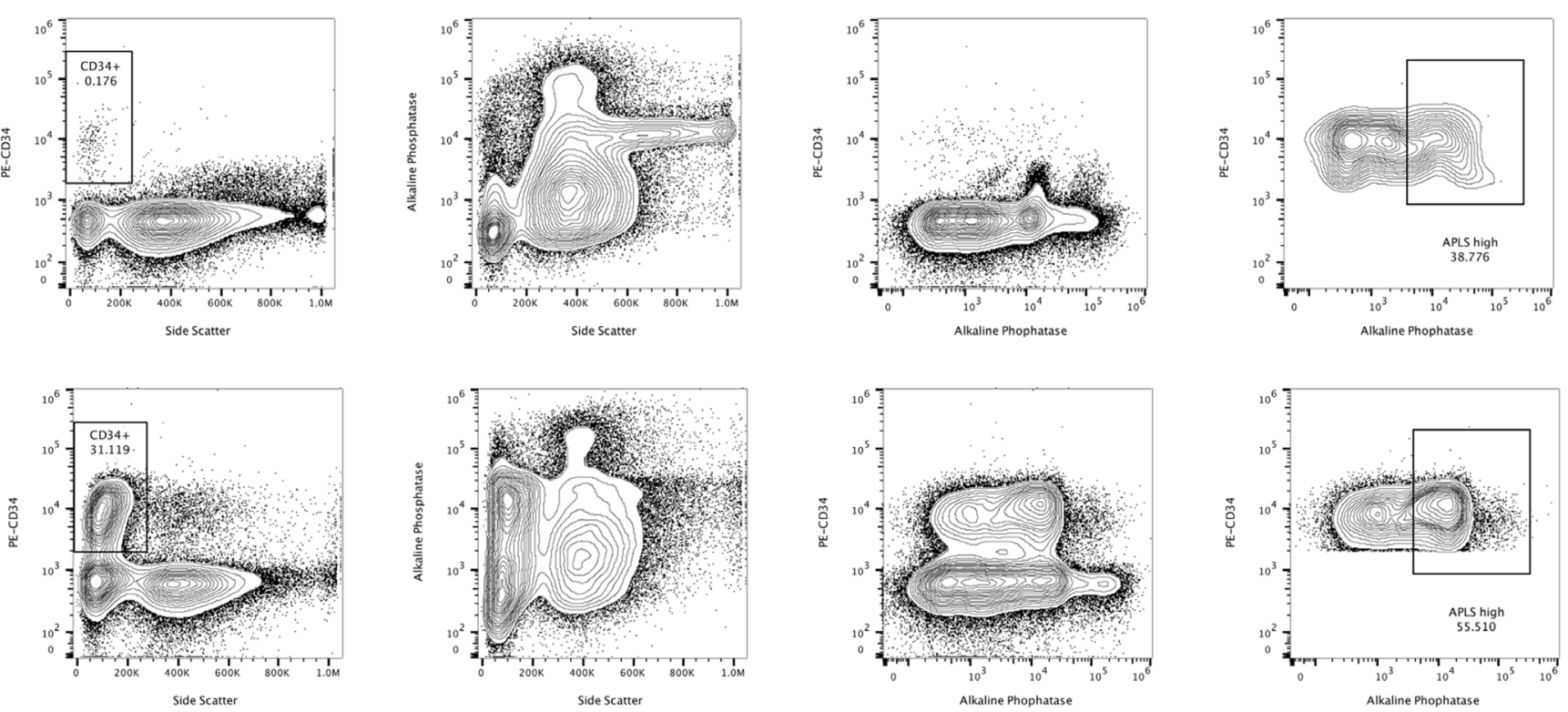

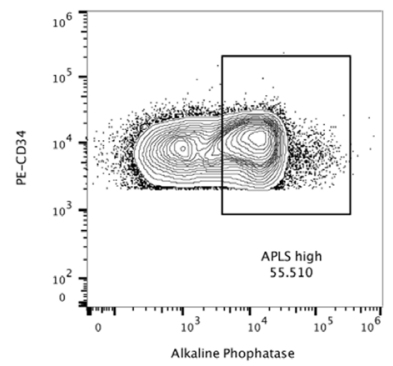

Figure 4: Representative flow cytometric study of the alkaline phosphatase activity in a patient of the ALP $\geq 12 \%$ group. Alkaline phosphatase positive cells are represented in combination with CD34 staining at diagnosis (upper row) and after relapse (lower row). Reference contour plots for two bone marrow aspirates are compared in the same patient, displaying high levels of alkaline phosphatase activity in combination with CD34 staining. The statistics in the region represents percentage of the gate. 
their informed consent in accordance with the Declaration of Helsinki. All procedures were in accordance with the internal protocols of our laboratory, which were authorized by the HGTiP Clinical Investigation Ethical Committee, in agreement with current Spanish legislation.

\section{Flow cytometry methods}

All EDTA-anticoagulated bone marrow $(n=38)$ and blood $(n=5)$ samples were prepared using a modified previously developed method [27] aimed at avoiding the lysis of erythrocytes during sample preparation, which can result in unwanted damage to leukocytes, and conceivably to leukemic cells. As most enzyme functions are performed at $37^{\circ} \mathrm{C}$, we first established an optimal Alkaline Phosphatase Live Stain (APLS, Thermo Fisher Scientific) stable loading time $(\mathrm{t}=20 \mathrm{~min})$ and temperature $\left(\mathrm{T}=37^{\circ} \mathrm{C}\right)$ to measure ALP enzymatic activity [14]. This dye is a cell-permeable fluorescent substrate for ALP that is non-toxic to cells. Importantly, ammonium chloride- and paraformaldehyde-based lysing solutions impair and almost completely abrogate ALP staining, are not recommended for detection of ALP + cells (Supplementary Figure 1). The KG-1a cell line, derived from Human Caucasian bone marrow acute myelogenous leukemia [31], was used as a positive control for highly expressing ALP+ cells (Supplementary Figure 2).

Briefly, our staining strategy used Vybrant ${ }^{\mathrm{TM}}$ DyeCycle ${ }^{\mathrm{TM}}$ Violet stain (DCV, Thermo Fisher Scientific), a low cytotoxicity permeable DNA-specific dye that can be used for DNA content cell cycle and stem cell Side Population analysis by flow cytometry [32]. DCV can be excited with violet $405 \mathrm{~nm}$ laser light and can be used for simultaneous measurement with APLS, which is excited with blue $488 \mathrm{~nm}$ laser light and its emission can be collected using a standard FITC filter (for example 530/30 $\mathrm{nm}$ ). Subsequently, cells were stained with fluorophoreconjugated monoclonal antibodies for $20 \mathrm{~min}$ at room temperature as recommended by the manufacturer (PECD123, PECy5-CD34 and PECy7-CD117, Sysmex $\mathrm{GmbH})$. The improved phycoerythrin (PE) signal using $561 \mathrm{~nm}$ excitation together with the fact that there is no need for color compensation between FITC and $\mathrm{PE}$ under $488 \mathrm{~nm}$ and $561 \mathrm{~nm}$ excitation, results in the improved immunophenotypic analysis of leukemic cells by multicolor flow cytometry [33].

Stained cells were diluted with Hank's Balanced Salt Solution (HBSS) (1000 $\mu \mathrm{L}$ final volume) prior to sample acquisition. All cell measurements were done using the Attune ${ }^{\mathrm{TM}}$ Acoustic Focusing Cytometer and the Attune $^{\mathrm{TM}}$ NxT Acoustic Focusing Cytometer (Thermo Fisher). Samples were acquired at $25-100 \mu \mathrm{L} / \mathrm{min}$ sample rates, and a minimum of 100,000 DCV+ events were collected per sample when possible. Threshold levels were set empirically using a Violet-Side Scatter (SSC) vs. DCV-H dual parameter plot to eliminate debris and the large numbers of red blood cells that are found in unlysed blood or bone marrow from detection. DCV was excited at $405 \mathrm{~nm}$ and its emission was collected using the following filter combination: 413 LP, 495 DLP, and 440/50 BP in the VL2 detector.

SSC was detected using the violet laser $405 \mathrm{~nm}$ with a 405/10 nm bandpass filter. APLS was detected with the blue laser $488 \mathrm{~nm}$ excitation and a 530/30 nm bandpass filter in the BL1 (Blue Laser) detector. For the Attune ${ }^{\mathrm{TM}}$ NxT upgraded with the yellow laser kit, PE was excited at $561 \mathrm{~nm}$ and its emission was collected using the following filter combination: 595 LP, $600 \mathrm{DLP}$, and 585/16 BP in the YL1 detector. PECy5 was detected with the yellow laser $561 \mathrm{~nm}$ excitation and a $695 / 40 \mathrm{~nm}$ bandpass filter in the YL3 detector. PECy7 was detected with the yellow laser 561 $\mathrm{nm}$ excitation and a 780/60 $\mathrm{nm}$ bandpass filter in the YL4 detector. APLS, PE, PECy5, PECy7 and DCV fluorescence are displayed on a logarithmic scale. Representative contour plot analysis for two bone marrow aspirates at diagnosis and relapse, used to calculate ALP+ cell numbers in combination with CD34 staining is shown in Figure 4.

ALP + cell numbers were determined in parallel to flow cytometry immunophenotyping of leukemia cells performed at the HGTiP clinical hematology laboratory. Standard immunophenotyping was performed on a FC500 (Beckman Coulter) flow cytometer until October 2016, and on a Navios ${ }^{\mathrm{TM}}$ flow cytometer (Beckman Coulter) from this date onwards. Blast cells were identified using cell surface protein markers according to the ELN recommendations [16]. MPO/CD45/CD34/ CD117/CD123/Myeloid/T-Lymphoid/B-lymphoid and megakaryocyte (MK) markers were used for typifying the blastic population through the identification of LAIPs (whenever possible), or through the detection of abnormalities in the different-from-normal cellular distribution in association with a low expression of CD45 and low SSC. A minimum of 20,000 events were studied at diagnosis. A marker was considered as positive when was expressed by $\geq 20 \%$ of blastic cells. Assessment of MRD was performed on bone marrow aspirates with a minimum sensitivity of $0.1 \%$. LAIPs identified at diagnosis were carefully looked for, and shifts in the relative scatter distribution of the different-from-normal pattern were also taken into account.

\section{Statistical methods}

We used receiver operating characteristic (ROC) analysis, area under the curve (AUC), sensitivity and specificity to measure prognostic accuracy of our test in predicting EFS. The optimal cut-off value was identified according to the Youden's Index to classify patients into two groups [34]. Differences between categorical variables in each group were compared using two-sided Fisher's exact tests. Differences between continuous variables in each group were compared using two-sided Wilcoxon 
rank sum tests. EFS and OS rates were estimated by the method described by Kaplan and Meier [35]. EFS was defined as the time from diagnosis until progression, death, or the last follow-up date. OS was defined as the time from diagnosis until death or the last follow-up date. Patients that underwent ASCT were censored. Differences in survival between each group were tested for statistical significance using the two-sided log-rank test with the Bonferroni method to adjust $P$ value. Univariate analysis using the Cox proportional hazards model was performed to investigate the impact of \% ALP at diagnosis on EFS and OS, adjusting for the following variables: age, sex, ELN prognostic-risk group, blast percentage, white blood cell (WBC) count and AML type (de novo vs, secondary). Factors prognostic for EFS and OS with a $P$-value $<0.5$ in the univariate analysis were studied in a multivariate analysis. A $P$-value $<0.05$ was considered statistically significant. R Studio version 1.1.463 (https://www.Rproject.org/) was used for all statistical analysis.

\section{CONCLUSIONS}

In this study we have found that increased cellular alkaline phosphatase activity in leukemic cells at diagnosis was significantly associated with a higher risk of relapse, or treatment resistance, and mortality. Due to the small number of patients enrolled in this study, this finding requires further investigation.

\section{Author contributions}

L.G.R., and J.P. performed the experiments. L.G.R., J.J., M.D.W., J.A.B., and J.P performed data interpretation, and drafted the manuscript. J.P. conceived of the study, participated in its design, conducted most experiments, and revised the manuscript.

\section{ACKNOWLEDGMENTS AND FUNDING}

The authors are very grateful to Sara Vergara and Minerva Raya, for kindly providing samples used in this study. The authors are also very grateful for their advice and technical support to Sergio Ramón, Víctor Querol, Clara Streiff, Paola Paglia, Luca Tiengo and Lluís Sanz from Life Technologies for all his comments and discussions on earlier work in this research field. Jordi Petriz also acknowledges the financial support from The Obra Social La Caixa-Fundació Josep Carreras and The Generalitat de Catalunya.

\section{CONFLICTS OF INTEREST}

M.D.W. and J.A.B. work for Thermo Fisher Scientific, which is in the business of selling flow cytometers and flow cytometry reagents.

\section{REFERENCES}

1. Cossarizza A, Chang HD, Radbruch A, Akdis M, Andrä I, Annunziato F, Bacher P, Barnaba V, Battistini L, Bauer WM, Baumgart S, Becher B, Beisker W, et al. Guidelines for the use of flow cytometry and cell sorting in immunological studies. Eur J Immunol. 2017; 47:1584-797. https://doi. org/10.1002/eji.201646632. [PubMed]

2. Ley TJ, Miller C, Ding L, Raphael BJ, Mungall AJ, Robertson A, Hoadley K, Triche TJ Jr, Laird PW, Baty JD, Fulton LL, Fulton R, Heath SE, et al, and Cancer Genome Atlas Research Network. Genomic and epigenomic landscapes of adult de novo acute myeloid leukemia. N Engl J Med. 2013; 368:2059-74. https://doi.org/10.1056/ NEJMoa1301689. [PubMed]

3. Papaemmanuil E, Gerstung M, Bullinger L, Gaidzik VI, Paschka P, Roberts ND, Potter NE, Heuser M, Thol F, Bolli N, Gundem G, Van Loo P, Martincorena I, et al. Genomic Classification and Prognosis in Acute Myeloid Leukemia. N Engl J Med. 2016; 374:2209-21. https://doi.org/10.1056/ NEJMoa1516192. [PubMed]

4. Döhner H, Weisdorf DJ, Bloomfield CD. Acute Myeloid Leukemia. N Engl J Med. 2015; 373:1136-52. https://doi. org/10.1056/NEJMra1406184. [PubMed]

5. Walter RB, Kantarjian HM, Huang $X$, Pierce SA, Sun Z, Gundacker HM, Ravandi F, Faderl SH, Tallman MS, Appelbaum FR, Estey EH. Effect of complete remission and responses less than complete remission on survival in acute myeloid leukemia: a combined Eastern Cooperative Oncology Group, Southwest Oncology Group, and M. D. Anderson Cancer Center Study. J Clin Oncol. 2010; 28:176671. https://doi.org/10.1200/JCO.2009.25.1066. [PubMed]

6. Seargeant LE, Stinson RA. Evidence that three structural genes code for human alkaline phosphatases. Nature. 1979; 281:152-54. https://doi.org/10.1038/281152a0. [PubMed]

7. McKenna MJ, Hamilton TA, Sussman HH. Comparison of human alkaline phosphatase isoenzymes. Structural evidence for three protein classes. Biochem J. 1979; 181:67-73. https://doi.org/10.1042/bj1810067. [PubMed]

8. Hass PE, Wada HG, Herman MM, Sussman HH. Alkaline phosphatase of mouse teratoma stem cells: immunochemical and structural evidence for its identity as a somatic gene product. Proc Natl Acad Sci U S A. 1979; 76:1164-68. https://doi.org/10.1073/pnas.76.3.1164. [PubMed]

9. Terao M, Mintz B. Cloning and characterization of a cDNA coding for mouse placental alkaline phosphatase. Proc Natl Acad Sci U S A. 1987; 84:7051-55. https://doi.org/10.1073/ pnas.84.20.7051. [PubMed]

10. Berstine EG, Hooper ML, Grandchamp S, Ephrussi B. Alkaline phosphatase activity in mouse teratoma. Proc Natl Acad Sci U S A. 1973; 70:3899-903. https://doi. org/10.1073/pnas.70.12.3899. [PubMed]

11. Takahashi K, Tanabe K, Ohnuki M, Narita M, Ichisaka T, Tomoda K, Yamanaka S. Induction of pluripotent stem cells 
from adult human fibroblasts by defined factors. Cell. 2007; 131:861-72. $\quad$ https://doi.org/10.1016/j.cell.2007.11.019. [PubMed]

12. Singh U, Quintanilla RH, Grecian S, Gee KR, Rao MS, Lakshmipathy U. Novel live alkaline phosphatase substrate for identification of pluripotent stem cells. Stem Cell Rev Rep. 2012; 8:1021-29. https://doi.org/10.1007/s12015-0129359-6. [PubMed]

13. Jordan CT, Guzman ML, Noble M. Cancer stem cells. N Engl J Med. 2006; 355:1253-61. https://doi.org/10.1056/ NEJMra061808. [PubMed]

14. Rico LG, Juncà J, Ward MD, Bradford J, Petriz J. Is alkaline phosphatase the smoking gun for highly refractory primitive leukemic cells? Oncotarget. 2016; 7:72057-66. https://doi. org/10.18632/oncotarget.12497. [PubMed]

15. Swerdlow SH, Campo E, Harris NL, Jaffe ES, Pileri SA, Stein $\mathrm{H}$, Thiele J, editors. WHO classification of tumours of haematopoietic and lymphoid tissues (Revised 4th edition). International agency for research on Cancer (IARC). Lyon; 2017. 4002 p.

16. Döhner H, Estey E, Grimwade D, Amadori S, Appelbaum FR, Büchner T, Dombret H, Ebert BL, Fenaux P, Larson RA, Levine RL, Lo-Coco F, Naoe T, et al. Diagnosis and management of AML in adults: 2017 ELN recommendations from an international expert panel. Blood. 2017; 129:424- 47. https://doi.org/10.1182/blood-2016-08-733196. [PubMed]

17. De Kouchkovsky I, Abdul-Hay M. 'Acute myeloid leukemia: a comprehensive review and 2016 update'. Blood Cancer J. 2016; 6:e441. https://doi.org/10.1038/bcj.2016.50. [PubMed]

18. Gupta S, Devidas M, Loh ML, Raetz EA, Chen S, Wang C, Brown P, Carroll AJ, Heerema NA, Gastier-Foster JM, Dunsmore KP, Larsen EC, Maloney KW, et al. Flow-cytometric vs. -morphologic assessment of remission in childhood acute lymphoblastic leukemia: a report from the Children's Oncology Group (COG). Leukemia. 2018; 32:1370-79. https://doi. org/10.1038/s41375-018-0039-7. [PubMed]

19. Ravandi F, Walter RB, Freeman SD. Evaluating measurable residual disease in acute myeloid leukemia. Blood Adv. 2018; 2:1356-66. https://doi.org/10.1182/ bloodadvances.2018016378. [PubMed]

20. Clarkson B, Fried J, Strife A, Sakai Y, Ota K, Okita T. Studies of cellular proliferation in human leukemia. 3. Behavior of leukemic cells in three adults with acute leukemia given continuous infusions of $3 \mathrm{H}$-thymidine for 8 or 10 days. Cancer. 1970; 25:1237-60. https:// doi.org/10.1002/1097-0142(197006)25:6<1237::AIDCNCR2820250602>3.0.CO;2-7. [PubMed]

21. Bonnet D, Dick JE. Human acute myeloid leukemia is organized as a hierarchy that originates from a primitive hematopoietic cell. Nat Med. 1997; 3:730-37. https://doi. org/10.1038/nm0797-730. [PubMed]

22. Blau HM, Brazelton TR, Weimann JM. The evolving concept of a stem cell: entity or function? Cell. 2001; 105:829-41. https://doi.org/10.1016/S0092-8674(01)00409-3. [PubMed]
23. Nguyen LV, Vanner R, Dirks P, Eaves CJ. Cancer stem cells: an evolving concept. Nat Rev Cancer. 2012; 12:133-43. https://doi.org/10.1038/nrc3184. [PubMed]

24. Stokol T, Schaefer DM, Shuman M, Belcher N, Dong L. Alkaline phosphatase is a useful cytochemical marker for the diagnosis of acute myelomonocytic and monocytic leukemia in the dog. Vet Clin Pathol. 2015; 44:79-93. https://doi.org/10.1111/vcp.12227. [PubMed]

25. Martí M, Mulero L, Pardo C, Morera C, Carrió M, Laricchia-Robbio L, Esteban CR, Izpisua Belmonte JC. Characterization of pluripotent stem cells. Nat Protoc. 2013; 8:223-53. https://doi.org/10.1038/nprot.2012.154. [PubMed]

26. Petriz J, Bradford JA, Ward MD. No lyse no wash flow cytometry for maximizing minimal sample preparation. Methods. 2018; 134-135:149-63. https://doi.org/10.1016/j. ymeth.2017.12.012. [PubMed]

27. Fornas O, Garcia J, Petriz J. Flow cytometry counting of CD34+ cells in whole blood. Nat Med. 2000; 6:833-36. https://doi.org/10.1038/77571. [PubMed]

28. Corti S, Locatelli F, Papadimitriou D, Donadoni C, Salani S, Del Bo R, Strazzer S, Bresolin N, Comi GP. Identification of a primitive brain-derived neural stem cell population based on aldehyde dehydrogenase activity. Stem Cells. 2006; 24:975-85. https://doi.org/10.1634/stemcells.2005-0217. [PubMed]

29. Ossenkoppele G, Schuurhuis GJ. MRD in AML: does it already guide therapy decision-making? Hematology Am Soc Hematol Educ Program. 2016; 2016:356-65. [PubMed]

30. Kuang Y, Miki K, Parr CJ, Hayashi K, Takei I, Li J, Iwasaki M, Nakagawa M, Yoshida Y, Saito H. Efficient, Selective Removal of Human Pluripotent Stem Cells via EctoAlkaline Phosphatase-Mediated Aggregation of Synthetic Peptides. Cell Chem Biol. 2017; 24:685-694.e4. https://doi. org/10.1016/j.chembiol.2017.04.010. [․ㅏbMed]

31. Koeffler HP, Billing R, Lusis AJ, Sparkes R, Golde DW. An undifferentiated variant derived from the human acute myelogenous leukemia cell line (KG-1). Blood. 1980; 56:265-73. https://doi.org/10.1182/blood.V56.2.265.265. [PubMed]

32. Petriz J. Flow cytometry of the side population (SP). Curr Protoc Cytom. 2013; Chapter 9:Unit9.23. https://doi. org/10.1002/0471142956.cy0923s64. [PubMed]

33. G Rico L, Juncà J, Ward MD, Bradford J, Petriz J. Yellowgreen laser-based flow cytometry for CD34+ progenitor cell counting. Cytometry A. 2018; 93:172-76. https://doi. org/10.1002/cyto.a.23299. [PubMed]

34. Youden WJ. Index for rating diagnostic tests. Cancer. 1950; 3:32-35. https://doi.org/10.1002/1097-0142(1950)3:1<32::AIDCNCR2820030106>3.0.CO;2-3. [PubMed]

35. Kaplan EL, Meier P. Nonparametric Estimation from Incomplete Observations. J Am Stat Assoc. 1958; 53:45781. https://doi.org/10.1080/01621459.1958.10501452. 